\title{
Two-level Osteotomy for Genu Valgum with Tibia Valga: Surgical Planning and Execution
}

\author{
Aarjav B. Naik ${ }^{1}$, Dhruv S. Shah ${ }^{2}$ \\ Learning Point of the Article: \\ Multiple deformities of the same limb should be recognized and managed as separate entities.
}

\section{Abstract}

Introduction: Proximal tibial injury leading to tibia valga which, in turn, leading to genu valgum is well described deformity. Management options vary between growth modulation and corrective osteotomy. Osteotomy which is used for skeletally mature patients can either be done in single stage or in multiple stages or in gradual manner.

Case Report: We describe here a method to obtain single-stage correction with two-level osteotomy in a 20-year-old patient. It includes medial closing wedge osteotomy at distal femur and translational osteotomy at tibial shaft.

Conclusion: This method of two-level osteotomy recognizes and treats two deformities (i.e., genu valgum and tibia valga) separately and achieves correction in one stage with perfect restoration of mechanical axis of lower limb and joint line obliquity of knee.

Keywords: Genu valgum, tibia valga, double-level osteotomy, closing wedge osteotomy, translational osteotomy.

\section{Introduction}

Tibia valga following proximal tibial metaphyseal injury is a common cause of genu valgum in children [1]. First such case was described by Lewis Cozen in 1953 [2]. Multiple theories were postulated over a period of time to explain its pathogenesis. At present, prevailing theory is growth stimulation of medial portion of proximal tibia due to fracture hyperemia. This theory is backed by two reports. Green reported that post-traumatic tibia valga was due to medial overgrowth of proximal tibia supported by finding of asymmetric growth arrest lines [3]. Zionts et al. demonstrated overactivity of medial tibial growth plate by radionucleotide scanning in similar setting [4]. Development of genu valgum after tibia valga can be explained by Wolff's law. Tibia valga shifts weight-bearing mechanical axis on the lateral side resulting in more compression on lateral half of distal femoral growth plate than medial half. This leads to more growth on the medial side than lateral side eventually leading to genu valgum. (Fig. 1) describes sequential progression of deformity. The purpose of this article is to describe various management options and describe in detail technique of two-level osteotomy for singlestage correction of deformity.

\section{Case Report}

A 20-year-old male patient presented with right-sided genu valgum deformity and $2 \mathrm{~cm}$ true shortening without DNVD. The patient had a history of trauma over the right knee 5 years ago which was managed conservatively with a splint. The patient noticed right knee deformity at the age of 16 years which progressed gradually until the age of 19 years. The deformity is non-progressive since then. The patient complained of occasional pain over knee. On examination, no tenderness over knee could be elicited and patellar tracking was normal. There was no ligamentous laxity around knee. His medical history and family history were unremarkable. The patient wanted correction of deformity and consented for surgery for same.

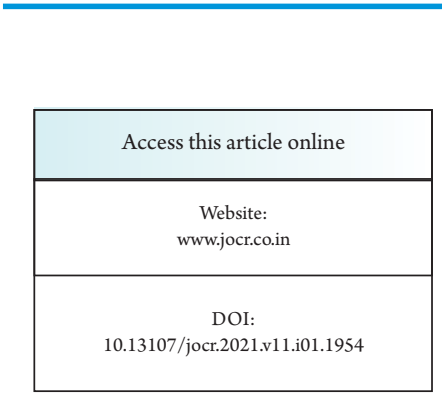

Journal of Orthopaedic This is an Open Access article distributed under the terms of the Creative Commons Attribution Non-Commercial License (http://creativecommons.org/licenses/by-nc/3.0) which permits unrestricted non-commercial use, distribution, and reproduction in any medium, provided the original work is properly cited.

\section{Author's Photo Gallery}

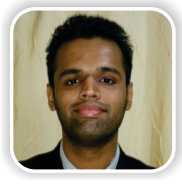

Dr. Aarjav B. Naik
${ }^{1}$ Department of Orthopedics, Government Medical College and New Civil Hospital, Surat, Gujarat, India,

${ }^{2}$ Department of Orthopedics, Baroda Medical College and SSG Hospital, Vadodara, Gujarat, India.

Address of Correspondence:

Dr. Aarjav B. Naik,

Department of Orthopedics, Government Medical College and New Civil Hospital, Surat - 395 001), Gujarat, India.

E-mail: naikaarjav@gmail.com 


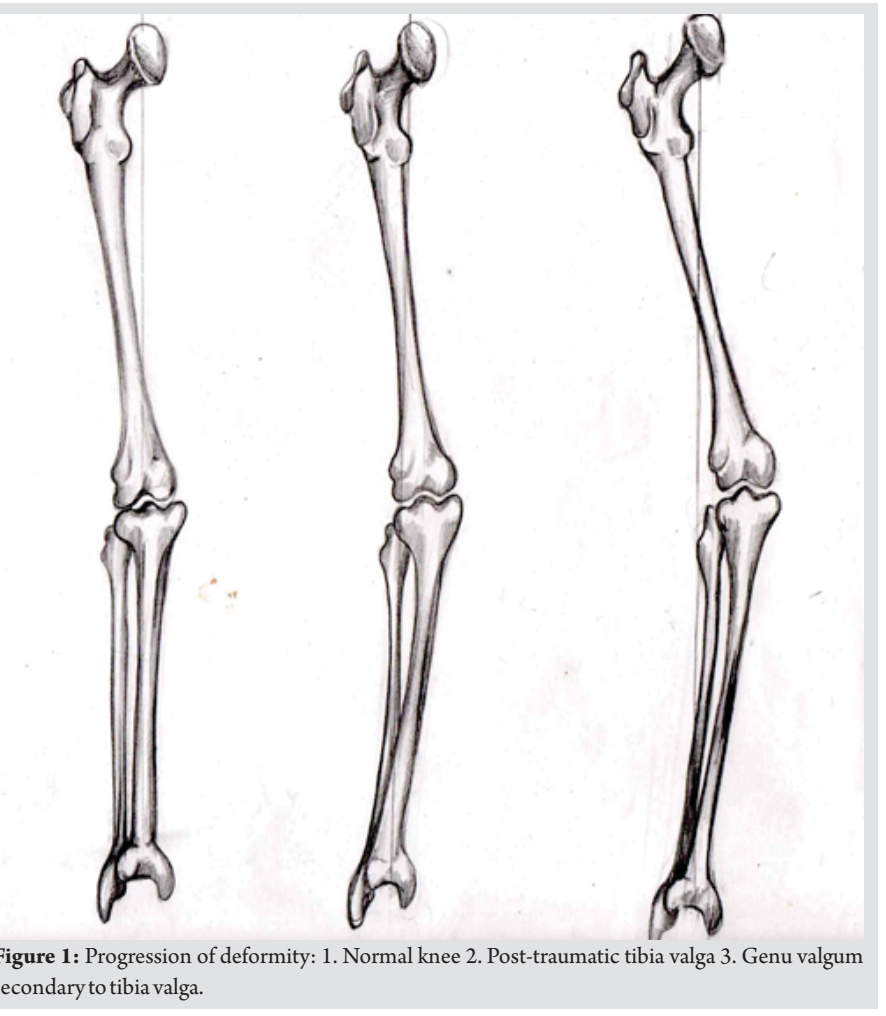

Specific pre-operative radiological investigations performed were standing bilateral lower limb scanogram for exact measurement of deformity (essential for bony deformities [5]) and magnetic resonance imaging (MRI) to evaluate both compartments of knee. (Fig. 2) shows pre-operative scanogram. On scanogram, angles were calculated on computer software to avoid manual error and to take account of magnification on the film. Lateral distal femoral angle was $67^{\circ}$ with knee joint line obliquity of $7^{\circ}$ varus which is abnormal. Joint line obliquity on opposite side was $3^{\circ}$ varus. When adjusted for joint line obliquity, genu valgum deformity turned out to be $16^{\circ}$ at the level of distal femur. Usually, genu valgum occurs due to distal femoral malalignment and should be corrected with distal femoral osteotomy while genu varum occurs due to proximal tibial malalignment and should be corrected with high tibia osteotomy [6]. Medial proximal tibial angle could not be calculated accurately because of bowing of tibial shaft. Tibial bowing was considered as multiapical deformity and total deformity of tibial shaft turned out to be $6^{\circ}$. This gives total deformity of $22^{\circ}$. There was $2 \mathrm{~cm}$ of true shortening as well. Center of rotation of angulation (CORA) of bowing deformity of tibial shaft was found at the level of junction of upper third and middle third of shaft of tibia. There is marked mechanical axis deviation (MAD) to lateral side. Neck shaft angles were $130^{\circ}$ on the right side and $135^{\circ}$ on the left side. No deformity was noted in sagittal plane. MRI revealed normal lateral compartment, medial compartment and other structures of knee obviating need of diagnostic arthroscopy.

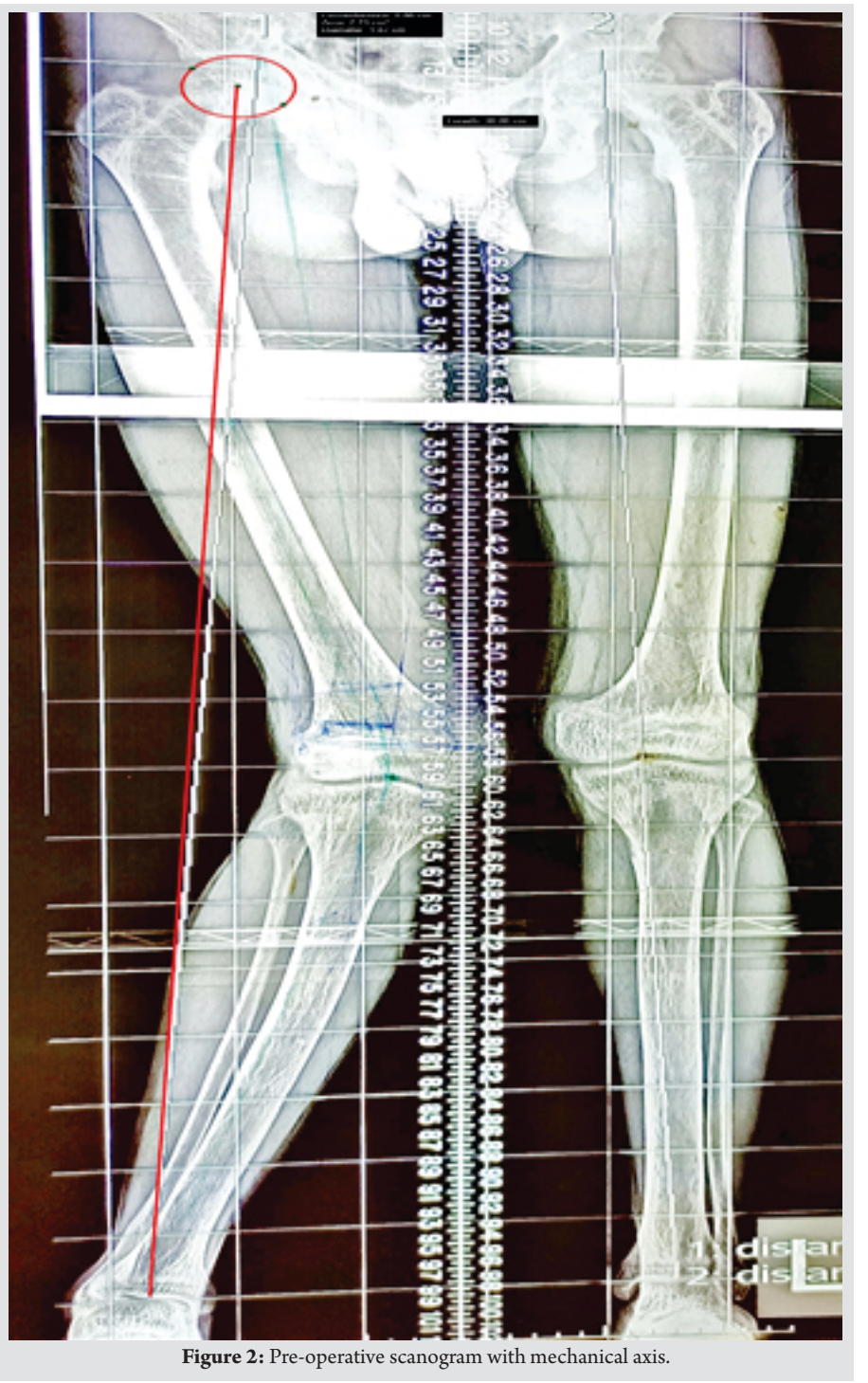

Goals of correction were to restore mechanical axis of lower limb while maintaining knee joint line obliquity ( $3^{\circ}$ varus) and to achieve single-stage correction without neurovascular injury. Total genu valgum deformity $\left(22^{\circ}\right)$ was divided into two components: Distal femoral component $\left(16^{\circ}\right)$ and tibial shaft component $\left(6^{\circ}\right)$. Surgical plan was devised for a single-stage correction utilizing medial distal femoral closing wedge osteotomy with translational osteotomy at CORA of shaft tibia. Closing wedge osteotomy was chosen over opening wedge due to higher biomechanical stability and scope of allowing early weight-bearing [6]. (Fig. 3) shows planned osteotomy.

Medial distal femoral closing wedge osteotomy was done using medial approach to distal femur. Initial $2 \mathrm{~K}$-wires were placed exactly parallel to knee joint line at metaphysis anteriorly and posteriorly. Perfect parallel placement of K-wires prevents flexion or extension deformity. Mediolateral diameter of osteotomy was calculated from these $\mathrm{K}$-wires using subtraction method.

(Tab. 1) Size of the wedge to be resected for desired correction 


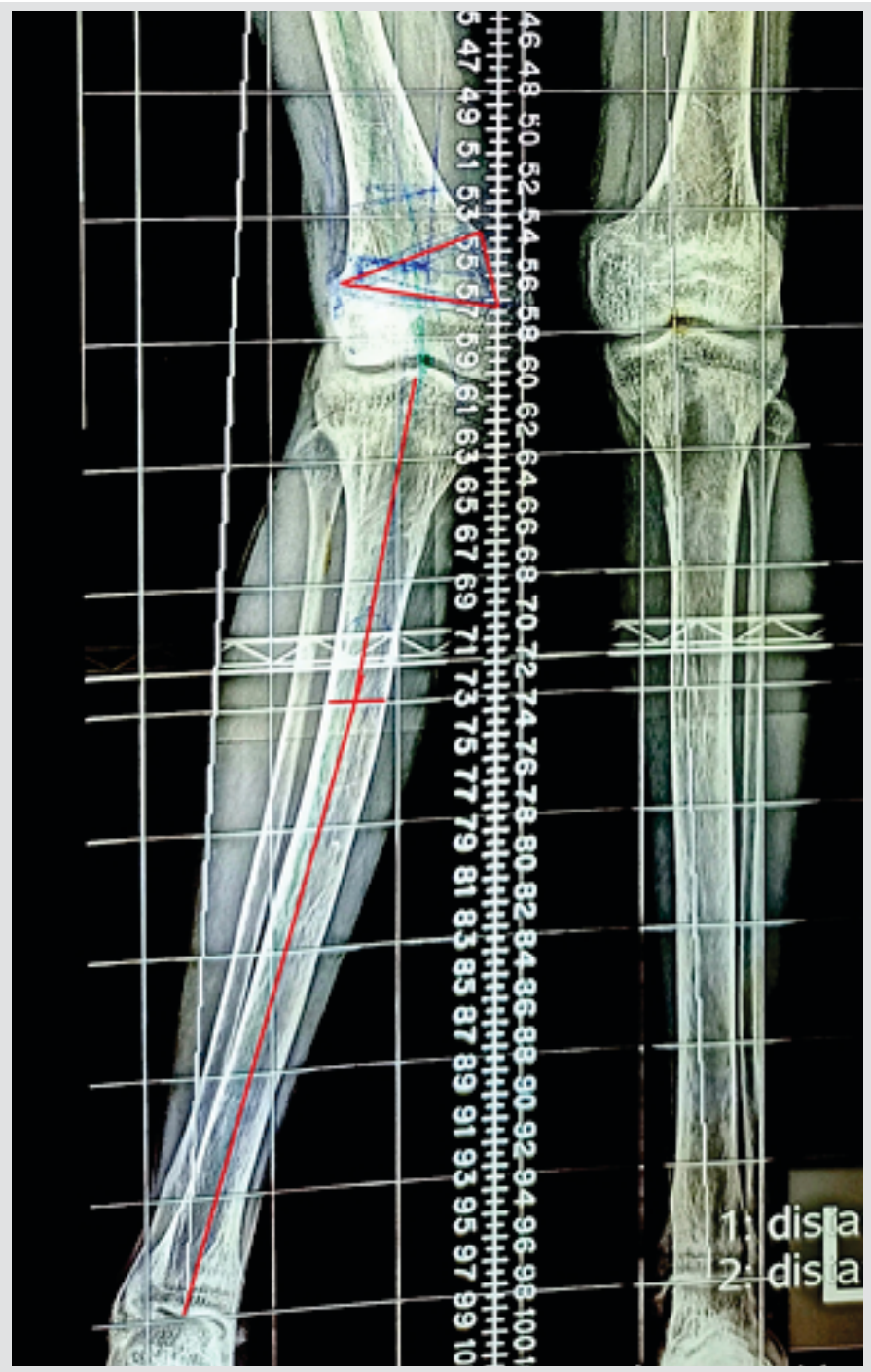

Figure 3: Planned osteotomy.

in $\mathrm{mm}$.

Size of the wedge to be resected was calculated from trigonometric table devised by Wylie et al. [6]. This table has inputs in form of mediolateral diameter of osteotomy and degrees of correction required (Table 1). Once the size of wedge was calculated, the second pair of K-wires was placed in convergent fashion after measuring the medial base with scale. Blunt Hohmann retractors were placed anteriorly and posteriorly to prevent injury to quadriceps anteriorly and neurovascular structures posteriorly. Osteotomy was carried out using oscillating saw using K-wires as guide. Specific care was taken to stop osteotomy $10 \mathrm{~mm}$ before lateral cortex to maintain lateral bony hinge and improve inherent stability of construct. Furthermore, completion of osteotomy at posterior cortex was done with osteotome to prevent injury to neurovascular structures. Bony wedge was removed and osteotomy was closed gently. Correction was checked by interoperative c-arm imaging using cautery wire. Osteotomy was fixed using $4.5 \mathrm{~mm}$ locking compression plate placed on anteromedial aspect of distal femur under direct visualization.

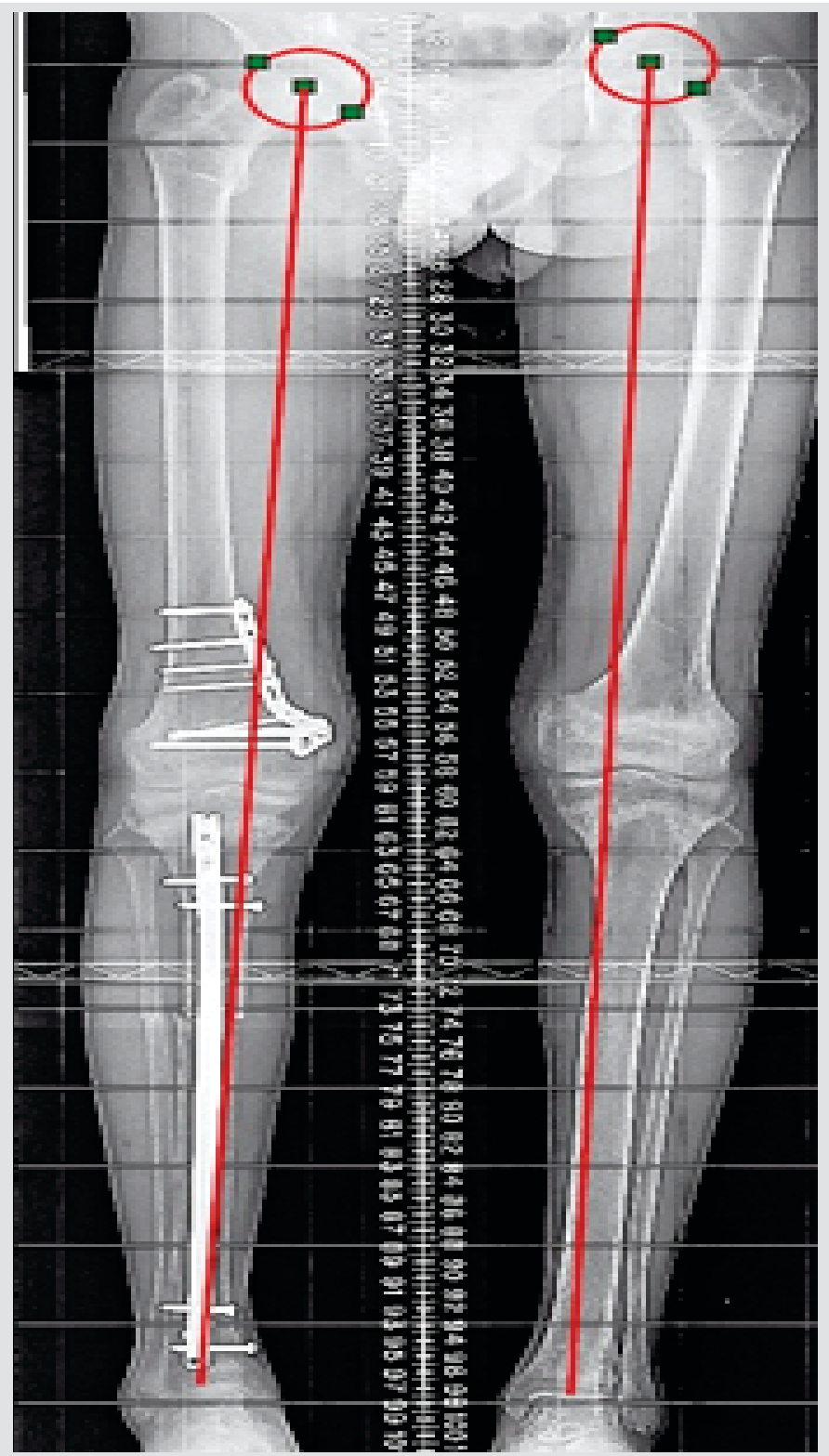

Figure 4: Post-operative scanogram with mechanical axis.

Translational osteotomy of tibia was done at CORA followed by internal fixation with tibia interlocked nail. Specific care was taken to prevent eccentric placement of nail after osteotomy which may lead to suboptimal alignment. First, tibial entry was taken with awl and guidewire was placed centrally in tibial canal. Progressive reaming was done over guidewire which created a centralized track for nail. Under fluoroscopic control, tibial osteotomy was completed with oscillating saw. Curved Hohmann's retractors were placed around tibia to prevent iatrogenic tissue injury. Osteotomy of fibula at same level was achieved manually with osteotomes using mini-open method. Tibia interlock nail was introduced over guidewire and locking was done. This technique ensures adequate correction of tibia valga due to central placement of a straight nail in the bone. Final alignment was checked using cautery wire method which showed perfect restoration of mechanical axis. Fig. 4 shows 
immediate post-operative scanogram.

\section{Discussion}

There are two types of methods for surgical correction of angular deformity: Growth modulation and corrective osteotomy.

Growth modulation corrects angular deformity by utilizing growth potential of physis and selectively inhibiting linear growth of hemiphysis. It is required that skeletal growth is remaining at the time of intervention and intervention is done at physis which contributes major part in skeletal growth of limb (distal femur and proximal tibia for lower limb).

Surgical hemiepiphysiodesis is a procedure, whereby we destroy hemiphysis surgically either through open [7] or percutaneous approach [8]. It cannot be reversed. It is critical to do this when patient is nearing skeletal maturity to avoid overcorrection by unabated growth of hemiphysis. This is called timed hemiepiphysiodesis. Green-Anderson growth charts and measurement of transverse width of physis are used to determine precise timing of hemiepiphysiodesis [9]. However, this method is not precise as there are inherent errors in determining skeletal age. To overcome this problem, untimed hemiepiphysiodesis [10] is used in which patient is followed up after intervention regularly and if correction is completed and growth remains, epiphysiodesis of remaining half of physis is completed. However, the problem of limb length discrepancy and compliance to follow-up remains with this method.

Hemiepiphysiodesis using staples or plate is a reversible procedure. It is utilized when significant skeletal growth is remaining (boys $<12$ years and girls $<10$ years). It is attractive because correction can be achieved soon after diagnosis of deformity rather than delaying until near skeletal maturity. Furthermore, once correction is achieved, implants can be removed allowing normal growth. Blount recommended that deformity should be overcorrected slightly as there is tendency of original deformity to rebound after removal of staples [11].

Corrective osteotomy is utilized once skeletal maturity is achieved. This utilizes removal or addition of wedge-shaped bone pieces based on trigonometric calculations to correct angular deformity. They are called closing wedge and opening wedge osteotomy, respectively. Translational osteotomy is used for bowing deformities.

Correction can be achieved acutely or gradually. Acute correction is achieved by removing or grafting a bone wedge surgically or by transverse osteotomy and fixing it with internal or external fixation devices. This usually causes higher softtissue violation, post-operative pain [12] comparing to gradual correction. Other risks are of compartment syndrome, neurovascular injury (more important for proximal tibial osteotomy [13]), and non-union. Gradual correction is usually achieved with osteotomy followed by gradual distraction overtime using external fixation device (Ilizarov ring fixator or hexapod etc.).

Deformity in this case has two components: Distal femur and shaft tibia. If correction is done with single-level osteotomy at distal femur, then it results in abnormal knee joint line obliquity and mechanical axis malalignment with mechanical axis passing through medial compartment of knee and not tibial spine which can lead to medial compartment arthritis in long term. Importance of the maintenance of neutral mechanical axis and knee joint line obliquity for better long-term outcomes has been well described in setting of osteoarthritis of knee [14]. The same principles can be applied in this setting. Double-level osteotomy solves this problem as it addresses both components separately and results in perfect restoration of joint line obliquity and mechanical axis.

For wedge osteotomies, either subtraction or addition type, calculation of wedge is based on trigonometric calculations which is an accurate method [15]. This method works best for uniapical deformities. For multiapical deformities like tibial bowing, there are two options: Either do a wedge osteotomy for each apex or do a translational osteotomy at level of CORA (center of rotation of angulation). Restoration of MAD happens with both options. Translational osteotomy has advantages over wedge osteotomy as it is less invasive and requires osteotomy at one level only as compared to the former. Thus, we have utilized translational osteotomy. CORA is defined as point of intersection of lines parallel to longitudinal axis of proximal and distal diaphyseal axis of the bone [15].

Table 1: Size of the wedge to be resected for desired correction in $\mathrm{mm}$

\begin{tabular}{|c|c|c|c|c|c|c|c|c|c|c|c|c|c|c|c|c|}
\hline \multirow{2}{*}{$\begin{array}{c}\text { Mediolateral Diameter } \\
\text { of Osteotomy }\end{array}$} & \multicolumn{16}{|c|}{ Preoperatively Measured Correction Angle } \\
\hline & $4^{\circ}$ & $5^{\circ}$ & $6^{\circ}$ & $7^{\circ}$ & $8^{\circ}$ & $9^{\circ}$ & $10^{\circ}$ & $11^{\circ}$ & $12^{\circ}$ & $13^{\circ}$ & $14^{\circ}$ & $15^{\circ}$ & $16^{\circ}$ & $17^{\circ}$ & $18^{\circ}$ & $19^{\circ}$ \\
\hline $50 \mathrm{~mm}$ & 3 & 4 & 5 & 6 & 7 & 8 & 9 & 10 & 10 & 11 & 12 & 13 & 14 & 15 & 16 & 16 \\
\hline $55 \mathrm{~mm}$ & 4 & 5 & 6 & 7 & 8 & 9 & 10 & 10 & 11 & 12 & 13 & 14 & 15 & 16 & 17 & 18 \\
\hline $60 \mathrm{~mm}$ & 4 & 5 & 6 & 7 & 8 & 9 & 10 & 11 & 12 & 14 & 15 & 16 & 17 & 18 & 19 & 20 \\
\hline $65 \mathrm{~mm}$ & 5 & 6 & 7 & 8 & 9 & 10 & 11 & 12 & 14 & 15 & 16 & 17 & 18 & 19 & 20 & 21 \\
\hline $70 \mathrm{~mm}$ & 5 & 6 & 7 & 8 & 10 & 11 & 12 & 13 & 15 & 16 & 17 & 18 & 20 & 21 & 22 & 23 \\
\hline $75 \mathrm{~mm}$ & 5 & 6 & 8 & 9 & 10 & 12 & 13 & 14 & 16 & 17 & 18 & 20 & 21 & 22 & 24 & 25 \\
\hline $80 \mathrm{~mm}$ & 6 & 7 & 8 & 10 & 11 & 13 & 14 & 15 & 17 & 18 & 19 & 21 & 22 & 24 & 25 & 26 \\
\hline
\end{tabular}


As with any surgical procedure, complications can occur in this procedure. Complications that are reported in literature are infection, thrombophlebitis, iatrogenic fracture, neurovascular injury, under correction, post-operative loss of correction, delayed union, and non-union. As with present case, hardware prominence was only complication encountered. The patient was given shoe raise of $2 \mathrm{~cm}$ as shortening persisted after surgery.

\section{Conclusion}

Post-traumatic tibia valga leading to genu valgum is a welldescribed deformity. This article discusses its management with one-stage double-level osteotomy after skeletal maturity. With proper pre-operative planning, full correction can be achieved. It is of paramount importance to recognize two components of deformity separately and address them separately to achieve total correction of mechanical axis and restoration of joint line obliquity.

\section{Clinical Message}

If you encounter post-traumatic genu valgum deformity, look for tibia valga. If present, this both deformities should be recognized separately and managed accordingly with twolevel osteotomy.

\section{References}

1. White RG, Mencio GA. Genu valgum in children: Diagnostic and therapeutic alternatives. J Am Acad Orthop Surg $1995 ; 3: 275-83$.

2. Cozen L. Fracture of the proximal portion of the tibia in children followed by valgus deformity. Surg Gynecol Obstet 1953;97:183-8.

3. Green NE. Tibia valga caused by asymmetrical overgrowth following a non-displaced fracture of the proximal tibial metaphysis.J Pediatr Orthop 1983;3:235-7.

4. Zionts LE, Harcke HT, Brooks KM, MacEwen GD. Posttraumatic tibia valga: A case demonstrating asymmetric activity at the proximal growth plate on technetium bone scan.J Pediatr Orthop 1987;7:458-62.

5. Filho EL, Torres MR, Silva MR, Lima FR, Aguiar JL. Simplified calculation for corrective osteotomies of long bones. Acta Ortop Bras 2016;24:253-8.

6. Wylie JD, Maak TG. Medial closing-wedge distal femoral osteotomy for genu valgum with lateral compartment disease. Arthrosc Tech 2016;5:e1357-66.

7. Phemister DB. Operative arrestment of longitudinal growth of bones in the treatment of deformities. J Bone Joint Surg Am 1933;15:1-15.

8. Canale ST, Russell TA, Holcomb RL. Percutaneous epiphysiodesis: Experimental study and preliminary clinical results.J Pediatr Orthop 1986;6:150-6.

9. Bowen JR, Torres RR, Forlin E. Partial epiphysiodesis to address genu varum or genu valgum. J Pediatr Orthop 1992;12:359-64.

10. Ferrick MR, Birch JG, Albright M. Correction of nonBlount's angular knee deformity by permanent hemiepiphyseodesis. J Pediatr Orthop 2004;24:397-402.

11. Tachdjian's Pediatric Orthopedics. In: Disorders of Knee. 5thed., Ch.21.p. 736.

12. Steel HH, Sandrow RE, Sullivan PD. Complications of tibial osteotomy in children for genu varum or valgum. Evidence that neurological changes are due to ischemia. J Bone Joint Surg Am 1971;53:1629-35.

13. Pinkowski JL, Weiner DS. Complications in proximal tibial osteotomies in children with presentation of technique. J Pediatr Orthop 1995; 15:307-12.

14. Babis GC, An KN, Chao EY, Rand JA, Sim FH. Double level osteotomy of the knee: A method to retain joint-line obliquity. Clinical results. J Bone Joint Surg Am 2002;84:1380-8.

15. Paley D, Tetsworth K. Mechanical axis deviation of the lower limbs. Preoperative planning of multiapical frontal plane angular and bowing deformities of the femur and tibia. Clin Orthop Relat Res 1992;280:65-71.
Conflict of Interest: Nil

Source of Support: Nil

Consent: The authors confirm that informed consent was obtained

from the patient for publication of this case report

\section{How to Cite this Article}

NaikAB, Shah DS. Two-level Osteotomy for Genu Valgum with Tibia Valga: Surgical Planning and Execution. Journal of Orthopaedic Case Reports 2021 January; 11(1):33-37. 